\title{
Ramsay Hunt Syndrome Complicated by Meningoencephalitis and Radiologic findings: a Rare Case Report
}

Investigative Magnetic Resonance Imaging

\section{Case Report}

Received: November 16, 2018

Revised: December 3, 2018

Accepted: December 7, 2018

\section{Correspondence to:}

Donghoon Lee, M.D.

Department of Radiology,

Seoul Medical Center, 156

Sinnae-ro, Jungnang-gu, Seoul

02053, Korea.

Tel. +82-2-2276-7000

Fax. +82-2-2276-7093

E-mail:jnoon276@gmail.com

This is an Open Access article distributed under the terms of the Creative Commons Attribution Non-Commercial License (http://creativecommons.org/licenses/ by-nc/4.0/) which permits unrestricted non-commercial use, distribution, and reproduction in any medium, provided the original work is properly cited.

Copyright (C) 2019 Korean Society of Magnetic Resonance in Medicine (KSMRM)

\author{
Youdae Lee, Donghoon Lee \\ Department of Radiology, Seoul Medical Center, Seoul, Korea
}

Ramsay Hunt syndrome with the complication of encephalitis or meningoencephalitis is rarely reported and uncommon in immunocompetent patients. The radiological manifestations of such cases usually involve the cerebellum and brainstem or exhibit the absence of any abnormality. We report a case of a 78-year-old immunocompetent man hospitalized with Ramsay Hunt syndrome, who later developed meningoencephalitis. The cerebrospinal fluid-study excluded other causes of meningoencephalitis, and the clinical diagnosis indicated varicella zoster virus meningoencephalitis. Magnetic resonance imaging revealed increased signal intensities in the bilateral temporal lobe, midbrain, and pons on T2-weighted imaging, and T2 fluid attenuated inversion recovery and contralateral asymmetric pachymeningeal enhancement. Contrast-enhanced T1-weighted imaging revealed ipsilateral facial nerve enhancement.

Keywords: Varicella-zoster virus; Ramsay Hunt syndrome; Meningoencephalitis; Magnetic resonance imaging

\section{INTRODUCTION}

Reactivation of the varicella zoster virus (VZV) in the geniculate ganglion typically causes Ramsay Hunt syndrome, which manifests as otalgia, vesicles in the auditory canal, ipsilateral facial palsy, and other vestibulocochlear symptoms. There exist a few reports on rare neurologic complications, such as encephalitis or meningoencephalitis, commonly affecting the elderly and immunocompromised patients. About two-thirds of the patients with encephalitis of Ramsay Hunt syndrome show positive findings on magnetic resonance imaging (MRI), with typical effect on the brainstem, cerebellum, and rarely the parieto-temporal lobe (1). There are no reported cases with abnormal meningeal enhancement. Herein, we report a rare case of a patient who initially presented with Ramsay Hunt syndrome and later developed meningoencephalitis, with positive MRI findings of contralateral asymmetric meningeal enhancement, and involvement of the bilateral temporal lobe, cerebellum, midbrain, pons, and ipsilateral facial nerve. 


\section{CASE REPORT}

A 78-year-old man with pain in the left ear, left facial palsy, left-side hearing discomfort, and dizziness for over a period of four days visited our outpatient clinic. He had hypertension, hyperlipidemia, and dementia, and was immunocompetent. He had no previous history of headrelated surgery or trauma. Upon physical examination, we noted that his left auricle was swollen, vesicles were seen, and his external acoustic canal showed erosion, discharge, and crust. He was diagnosed with Ramsay Hunt syndrome and admitted for steroid and antiviral therapy. The treatment plan included administration of acyclovir (5 $\mathrm{mg} / \mathrm{kg}$ ) thrice daily and dexamethasone (total dose $60 \mathrm{mg}$ ) twice daily.

On the following day, the patient presented with worsening gait disturbance, headache, fever, swallowing discomfort, and neck stiffness. Additional spinal tapping and diffusion-weighted magnetic resonance (DW-MR) imaging were performed using a 1.5T MRI system (MAGNETOM Avanto, Siemens AG, Erlangen, Germany). Although DWMR did not show diffusion restriction, the apparent diffuse coefficient $(A D C)$ image revealed increased signal intensity in the left external ear and mastoid area (Fig. 1a, b). Furthermore, we reviewed DW-MR after performing follow up MRI. A mild increase in the signal intensities in midbrain and right medulla on b0 images was detected (Fig. 1c, d).

Analysis of the cerebrospinal fluid (CSF) showed an elevated white blood cell (WBC) count $(360 / \mu \mathrm{L}$, with $33 \%$ lymphocytes) and protein level (703.3 mg/dL). CSF culture, immunohistochemistry, and polymerase chain reaction (PCR) were performed to detect herpes simplex virus types

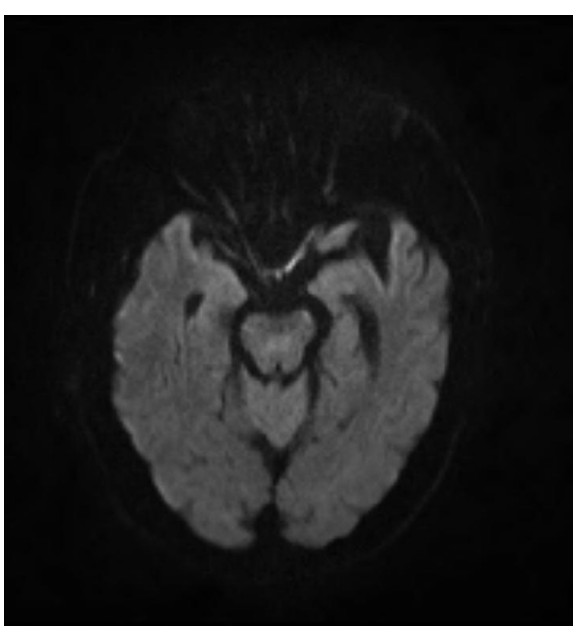

a

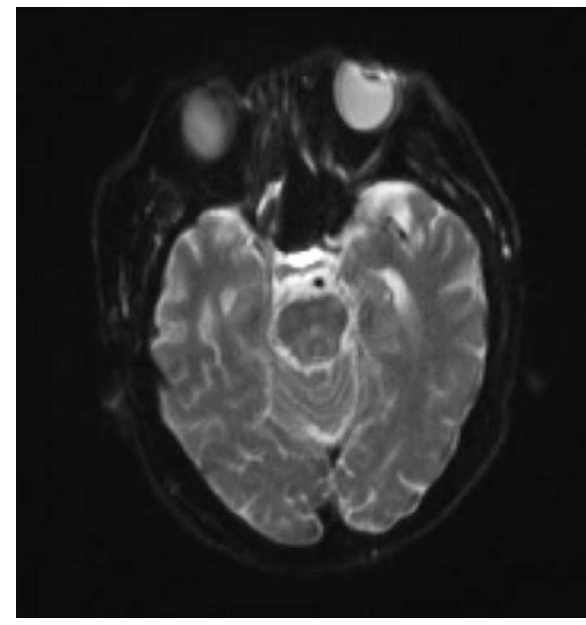

C

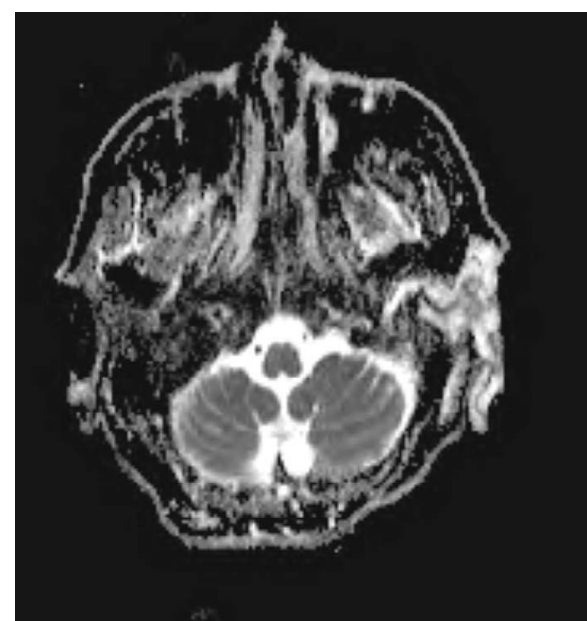

b

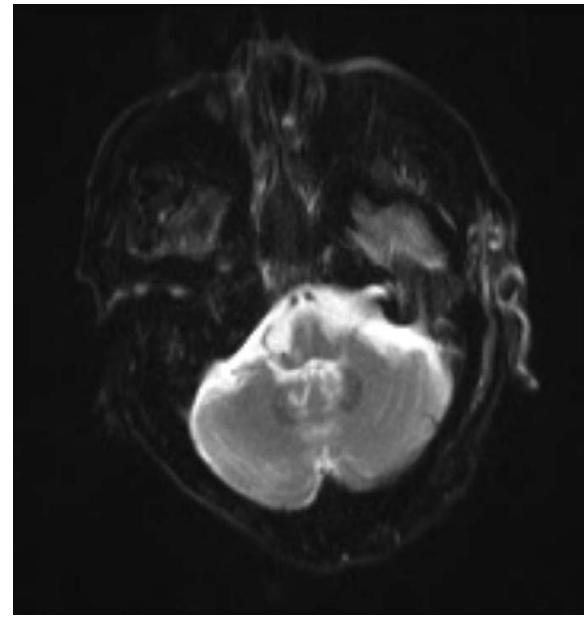

d
Fig. 1. (a, b) DW-MR (b1000) showing no diffusion restriction. $A D C$ image showing increased signal intensities in the left external ear and mastoid area. ( $c_{i}$ d) DW-MR (b0) showing a mild increase in the signal intensities in the midbrain and right medulla. 
1 and 2 (HSV-1/2), enterovirus, Epstein-Barr virus (EBV), cytomegalovirus (CMV), VZV, measles, mumps, tuberculosis, and other bacteria. While all culture and almost all PCR results were negative, the VZV PCR result was positive.

On the third day, follow-up MRI was performed using the 3T MRI system (Achieva, Philips Healthcare, Best, the Netherlands), with T2-weighted imaging (T2-WI) and T2 fluid attenuated inversion recovery (T2-FLAIR) showing increased signal intensities in the bilateral temporal lobe, midbrain, pons, cerebellum, and right medulla. Contrast- enhanced T1-WI (CE T1-WI) showed contralateral asymmetric pachymeningeal enhancement along the right cerebral convexity. In addition, there was an enhancement of left mastoid, external ear and facial nerve (Fig. 2).

The dose of acyclovir was increased to $10 \mathrm{mg} / \mathrm{kg}$ thrice daily under the assumption that progressive worsening was most likely to include neuritis and meningoencephalitis.

One week following admission, a follow-up CSF study

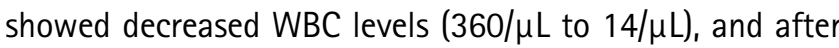
three weeks, improvement in the patient's symptoms was

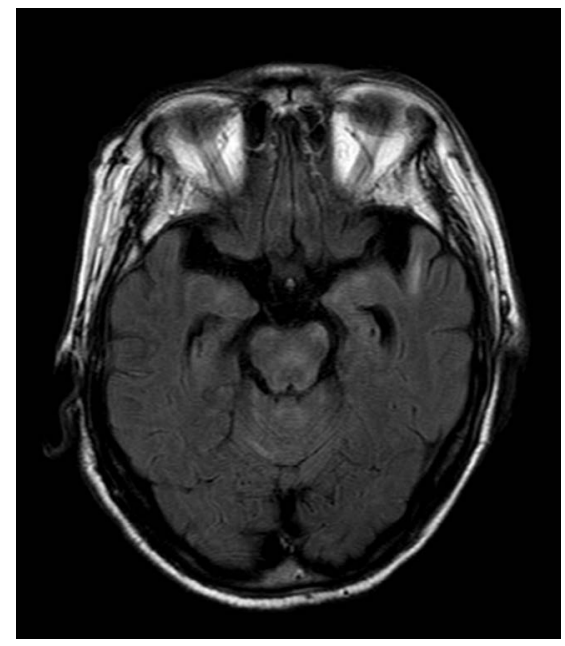

a

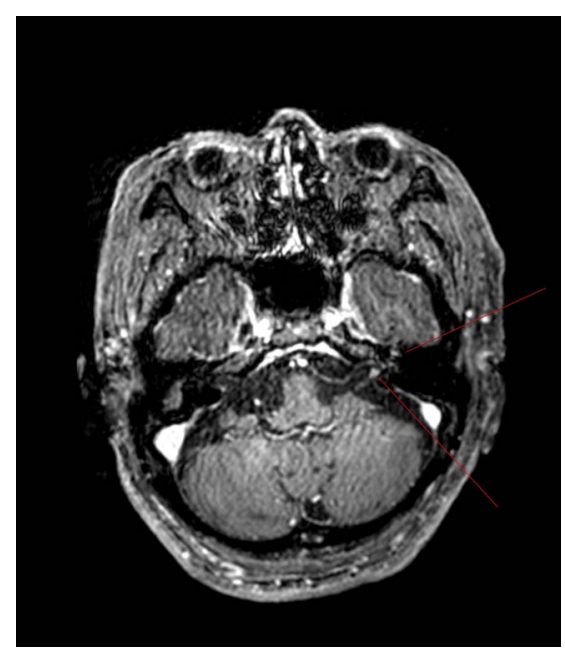

d

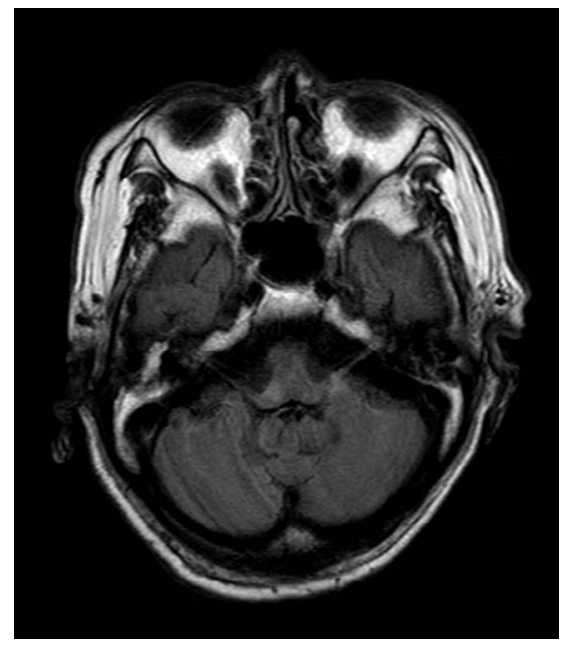

b

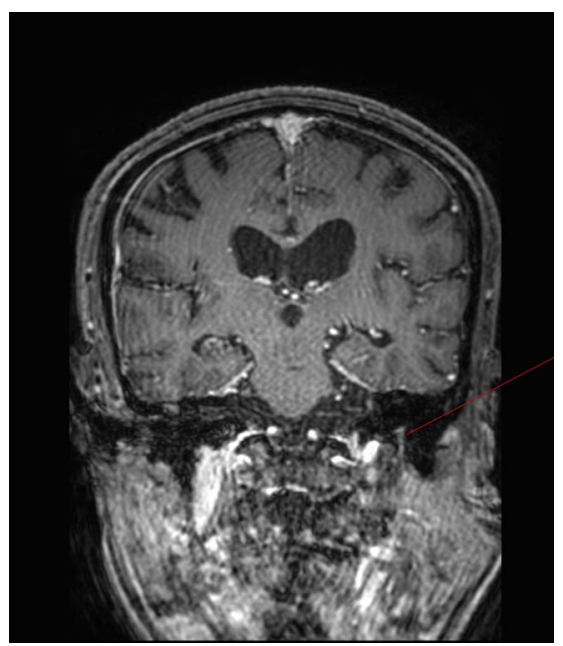

e

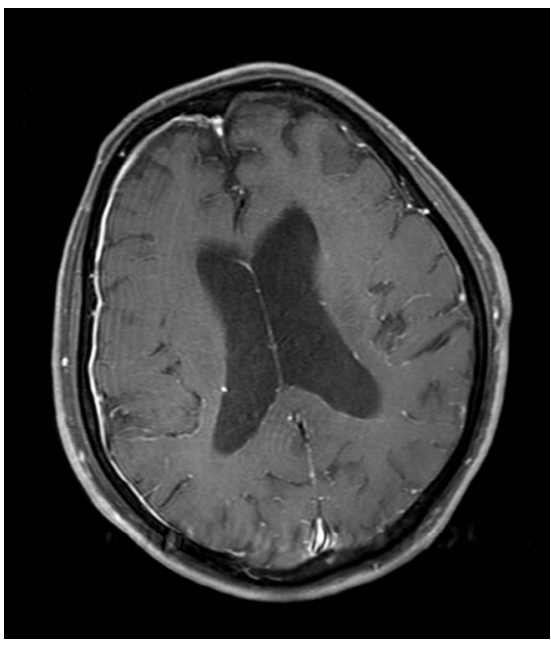

C

Fig. 2. T2-FLAIR image showing increased signal intensities in the bilateral temporal lobes, midbrain, and superior cerebellum. (a) T2-FLAIR image showing increased signal intensities in the bilateral temporal lobes, midbrain, and superior cerebellum. (b) T2-FLAIR image showing increased intensities in the right medulla and inferior portion of the left middle cerebellar peduncle. (c) CE T1-WI showing contralateral asymmetric pachymeningeal enhancement along the right cerebral convexity. $(d, e) C E T 1-W I$ showing the left facial nerve enhancement, involving the canalicular segment, labyrinthine segment, geniculate ganglion, tympanic segment (not shown), and mastoid segment. 
observed.

\section{DISCUSSION}

Ramsay Hunt syndrome is defined as VZV infection involving the sensory fibers of the facial and vestibulocochlear nerve, and may less commonly involve the glossopharyngeal and vagus nerves. The clinical symptoms include facial paralysis, erythematous vesicular rash on the ear or in the mouth, tinnitus, hearing loss, nausea, vomiting, vertigo, and nystagmus (2).

In rare cases, VZV can spread to the central nervous system in both immunocompetent and immunocompromised patients, being more frequent in the latter (3). The virus can spread in the centripetal, centrifugal, or both the patterns. In immunocompetent patients, it usually spreads in a centripetal pattern, into the proximal nerve roots adjacent to the dorsal root ganglia, causing neuritis, plexitis, radiculitis, or myelitis. In immunocompromised patients, VZV tends to be reactivated at multiple levels of dorsal root ganglia with subsequent dissemination. Therefore, patients are more widely and severely affected and exhibit higher morbidity and mortality rates $(3,4)$.

VZV has an additional feature of the ability to invade the blood vessels. In immunocompetent patients, the virus may spread to the large blood vessels at the base of the brain, causing inflammation of the vessel wall and ipsilateral brain infarction. There is great variability in the extent of inflammation in the affected blood vessels and though this is thought to be dependent on the onset of virus-mediated damage, it may also be due to other unknown factors. In the case of VZV, inflammation leads to meningoencephalitis, myelitis, neuritis, and large-vessel vasculopathy. In immunocompromised patients, VZV affects small blood vessels and cause small vessel vasculopathy, multifocal leukoencephalopathy and ependymitis (3).

The diagnosis of VZV-related encephalitis is typically based on a combination of clinical, laboratory, neuroimaging, and electrophysiological findings. The presence of fever, CSF pleocytosis, or changes in the MRI or electroencephalogram findings are indicative of encephalitis (5). PCR-based assays of CSF are the most rapid and sensitive method for diagnosing VZV-related neurological complications. In addition, the presence of a VZV-specific antibody or anti-VZV immunoglobulin M (IgM) antibody in the CSF is specific to the patients with encephalitis even when the PCR results are negative (6).
Radiologic findings of the common VZV neurologic complications are well known. VZV cerebellitis manifests as cerebellar cortical edema and swelling, VZV vasculitis as focal stenosis of major intracranial arteries, and VZV ischemic stroke most commonly involves the basal ganglia. Ramsay Hunt syndrome shows contrast enhancement in the internal auditory canal, membranous labyrinth, and intratemporal facial nerve (7).

Only a few cases of Ramsay Hunt syndrome with associated encephalitis are reported. Shen et al. (8) described a case of Ramsay Hunt syndrome complicated by brainstem encephalitis with positive MRI findings of T2-WI and FLAIR high signal intensity in the basal pons and medulla oblongata. Kaski et al. (9) reported a case of rhombencephalitis presenting as Ramsay Hunt syndrome with positive MRI findings of T2-WI and FLAIR high signal intensity in the pons, medulla, and floor of the fourth ventricle. Ricigliano et al. (1) reported an interesting case of a patient with Ramsay Hunt syndrome with clinically suspected encephalitis with CSF-positive findings but negative MRI findings. Ricigliano et al. (1) also revealed that $31 \%$ of Ramsay Hunt syndrome cases with encephalitis presented with negative MRI findings. In our literature search, almost all the patients with positive MRI findings showed ipsilateral involvement of the brain (if the Ramsay Hunt syndrome patients were affected on their left side, they exhibited left brain and brainstem involvement). Additionally, there are no published cases of diffuse meningeal enhancement detected with MRI. However, in our case study, the patient displayed involvement of the bilateral temporal lobe and cerebellum, and contralateral pachymeningeal enhancement. The radiological findings were non-specific for VZV meningoencephalitis; however, the clinical and laboratory findings indicated VZV infection and excluded other CSF infectious agents such as HSV, EBV or other bacteria/viruses.

In conclusion, we report a rare case of Ramsay Hunt syndrome in an immunocompetent patient with meningoencephalitis presenting with positive MRI findings of diffuse meningeal enhancement and involvement of the brain and facial nerve. Generally, diagnosis of Ramsay Hunt syndrome is based in a clinical setting and MRI is performed to check for neurological complications. This case report highlights that awareness of this unique manifestation will enable radiologists to diagnose Ramsay Hunt syndrome related to meningoencephalitis. 


\section{REFERENCES}

1. Ricigliano VAG, Saraceno L, Cavalli M, Rodegher M, Meola G. Slowly progressing varicella zoster brainstem encephalitis complicating Ramsay Hunt syndrome in an immunocompetent patient: case report and review of the literature. J Neurovirol 2017;23:922-928

2. Sweeney CJ, Gilden DH. Ramsay Hunt syndrome. J Neurol Neurosurg Psychiatry 2001;71:149-154

3. Kleinschmidt-DeMasters BK, Gilden DH. Varicella-Zoster virus infections of the nervous system: clinical and pathologic correlates. Arch Pathol Lab Med 2001;125:770780

4. Gilden D. Varicella zoster virus and central nervous system syndromes. Herpes 2004;11 Suppl 2:89A-94A
5. Halperin JJ. Diagnosis and management of acute encephalitis. Handb Clin Neurol 2017;140:337-347

6. Debiasi RL, Tyler KL. Molecular methods for diagnosis of viral encephalitis. Clin Microbiol Rev 2004;17:903-925, table of contents

7. Soares $B P$, Provenzale JM. Imaging of herpesvirus infections of the CNS. AJR Am J Roentgenol 2016;206:39-48

8. Shen YY, Dai TM, Liu HL, Wu W, Tu JL. Ramsay Hunt syndrome complicated by brainstem encephalitis in varicella-zoster virus infection. Chin Med J (Engl) 2015;128:3258-3259

9. Kaski D, Davies N, Seemungal BM. Varicella-zoster virus meningo-rhombencephalitis presenting as Ramsey Hunt. Neurology 2012;79:2291-2292 